\title{
Image Preprocessing Technology of Screen Coding Based on Compression Perception
}

\author{
Yanpeng Sun and Juan Wang \\ College of Electronics Information Engineering Shenyang Aerospace University, Shenyang 110136, China
}

\begin{abstract}
In this paper, a new preprocessing method is proposed to solve the problem that the image of the print screen coding image is not ideal because the information bit is small, the symbol is not clear and the noise is serious. This paper focuses on the algorithm of image filtering based on compression sensing and morphology and image skew correction based on Gabor wavelet. Compared with the traditional filtering, the combined filtering algorithm can reduce the noise while enhancing the protection of image feature information. The improved hough transform algorithm is also more suitable for coding image processing to achieve more accurate tilt correction. The experiment proves the superiority of the proposed algorithm.
\end{abstract}

Keywords - screen coding; compression perception; combinatorial filtering; morphology; tilt correction

\section{INTRODUCTION}

Screen coding technology has the advantages of large amount of hidden information, high security, low cost, good robustness and strong anti-attack ability ${ }^{[1]}$. It can not only replace QR code, watermarking technology, RFID technology, but also will realize the highly confidential communications of a combination of information encryption and information hiding ${ }^{[2]}$.In recent years, the field of paper media information security development get more and more attention. With the continuous development and improvement of technology, the screen coding develop toward the direction of the richer way of coding, carrier adaptability, better performance and wider application. Therefore, the automatic identification technology of screen coding has become the focus of research in the new period. As a key link in the identification of technology, coding image preprocessing technology research is imminent.

At present, the preprocessing of screen image is still in the traditional processing and ideal treatment. However, in the actual application process,it not only requires effective suppression of clutter while retaining the rich details, but also must take into account the capacity, storage space ,real-time and other issues. The traditional filtering methods are mostly based on the Nyquist theorem. That can not meet the requirements of coding image processing. A direct result of the screen coding application range is severely limited.

\section{Methodology}

\section{A. Compression Perception}

Compression-aware image processing mainly refers to the use of less sample data on the image signal reconstruction recovery. It's core: sparse representation of signals, observation matrix design and signal reconstruction algorithms. The signal is projected onto the orthogonal transform base using the sparse representation of the signal as a brief representation of the original signal, and then find an observation matrix satisfying the constraint equivalence condition with the transformation base. The technology of using signal compression to restore the signal is completely across the Nyquist sampling law constraints, reducing the sampling rate. The use of the sparsity of the signal not only greatly reduces the time required to obtain the image data but also saves the storage space. Specific theoretical basis and mathematical model shown in Figure 1:

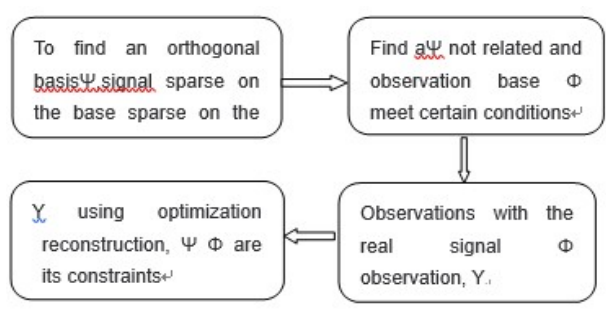

FIGURE I COMPRESSED AWARENESS PROCESS

Compressed Sensing Equation:

The original signal $X$ is represented by a linear combination of orthonormal basis: $\psi^{T}=\left[\psi_{1}, \psi_{2}, \ldots \psi_{k}, \ldots \psi_{K}\right]$.

$$
X=\sum_{\mathrm{k}=1}^{N} \psi_{k} s_{k}=\psi S
$$

Using the optimization method to recover $\mathrm{X}$ from the observation value $Y$ :

$$
Y=\phi X=\phi \psi S
$$

Where $S$ is the sparse coefficient.

Optimization:

$$
\min _{\alpha}\|\alpha\|_{l_{0}} \text { s.t } Y=\phi \psi \alpha
$$

Among them, under certain conditions L1 minimum norm and L0 minimum norm can be equivalent to replace, get the same solution.

$$
\min _{\alpha}\|\alpha\|_{l_{1}} \text { s.t } Y=\phi \psi \alpha
$$


Thus obtaining the estimate $\mathrm{S}^{\prime}$ of the sparse coefficient S.Then the original signal:

$$
x^{\prime}=\psi s^{\prime}
$$

The way of using reconstruction algorithm to solve the optimization problem should regard accurate reconstruction of the image as the primary factor. After that consider to reduce the image reconstruction time, to reduce the complexity of computing and other factors.

At present, several types of algorithms are proposed such as ACGP, MP, iterative threshold algorithm and combinatorial algorithm ${ }^{[3]}$. Among all algorithms, the iterative threshold algorithm is most suitable for coding image processing on the reconstruction algorithm requirements. The iterative threshold method is an alternative algorithm of greedy algorithm. It is generated by continuous projection and threshold. It can not only reduce the complexity of the algorithm, but also combine some additional optimization criteria. In this paper, we combine morphological filtering to improve image recovery quality.

\section{B. Morphological Algorithm}

Morphological processing of image refers to the analysis and processing of images by means of mathematical morphology. The main content is to explore the image using the structural elements, to get the structure of the image information, according to the relationship of structural elements and image filling, and making further processing. Structural elements selected arbitrariness, so that the morphological processing with a high degree of timeliness, the process is simple. That is consistent with the requirements of the screen coding in practical applications.

\section{A. Expansion and Corrosion}

Expansion operation:

$$
A \otimes S=\{(x, y) \mid(x, y) \in A, S(x, y) \cap A \neq \varnothing\}
$$

Where $\mathrm{A}$ is the target image and $\mathrm{S}$ is the structural element.

The expansion can be used to add any background pixels that are connected to any foreground pixel, increasing the size of the feature region or region.

Corrosion operation:

$$
A \Theta S=\{(x, y) \mid(x, y) \in A, S(x, y) / A=\varnothing\}
$$

Corrosion is to remove any foreground pixels that are connected to any of the background pixels. Corrosion can completely remove extraneous pixels that represent point noise or line defects.

\section{Open operation and Closed Operation}

Open operation: The target image is first subjected to corrosion treatment. And then use the expansion method to restore. With the $A \circ S$ expression.

$$
A \circ S=(A \Theta S) \otimes S
$$

It is possible to open the gap in which the feature is connected.

Closed operation: the target image to do the first expansion, and then corrosion treatment to restore. With the $A \bullet S$ expression.

$$
A \cdot S=(A \otimes S) \Theta S
$$

The closed operation is often used to connect and fill the gaps in the image features.

\section{High-hat filtering and low-hat filtering}

The operations are defined as:

$$
\begin{aligned}
& H=A-(A \circ S) \\
& H=A-(A \bullet S)
\end{aligned}
$$

Among them, the high-hat filtering operation can enhance the image contrast; low-hat filtering operation can get the edge of the image.

\section{The Tilt Correction Algorithm}

Tilt correction is mainly composed of three processes: boundary extraction, tilt angle detection, tilt correction.

Boundary extraction: Using the method of Gabor wavelet signature extraction make Gabor wavelet transform at a single scale with standard image by selecting the appropriate angle of Gabor wavelet.And than obtain the number of transform coefficients at different angles.At the same time, do the same transform to the oblique image .On this basis, the horizontal edge feature of the image is extracted.

Tilt angle detection: Hough algorithm is used to do Hough transform for tilt image to detect the tilt angle. The specific process is as follows:

(1) Assume that the image edge feature is $(d, \theta)$, then the coordinate transformation formula:

$$
x \cos \theta+y \sin \theta=d
$$

Where, $x, y$ represents the Cartesian coordinates of the standard image pixels. Set up a matrix of accumulator by the representation $A(d, \theta)$, and set all the elements in the matrix to 0 , select the pixels $\left(x^{\prime}, y^{\prime}\right)$ that are satisfied by the representation of the image $\theta=\theta^{\prime}$. Calculate the following formula

$$
x \cos \theta+y \sin \theta=d
$$


(2) in the correction process, $A(d, \theta)$ corresponding to a column that a representative of $\theta^{\prime}$, because there is a local maximum of $A(d, \theta)$, it can be set to a threshold value $\mathrm{T}$

$$
\mathrm{T}=\mathrm{V} \max \mathrm{A}(d, \theta)
$$

In the above equation, after satisfying the condition of $V \leq 1$, the Hough transform to $A(d, \theta)$ is made by the threshold value $T$

$$
\mathrm{A}(d, \theta)=0, \quad \text { if } \mathrm{A}(d, \theta) \leq T
$$

(3) In the correction process, the image tilt angle is obtained by performing column addition to $A(d, \theta)$.

\section{Tilt correction:}

In the correction process, according to gotten angle, the corrected image is obtained by the rotation function.

\section{SCREen Coding IMAge Preprocessing Framework}

Under the influence of scanning condition and random interference, the original image after scanning is difficult to identify directly, and that must be filtered, enhanced, corrected and other image processing process to read and decode. In this paper, the combinatorial filtering method based on compression perception ,morphological method and the improved Hough transform method are used as the preprocessing method. The preprocessing flow chart is shown in the figure.

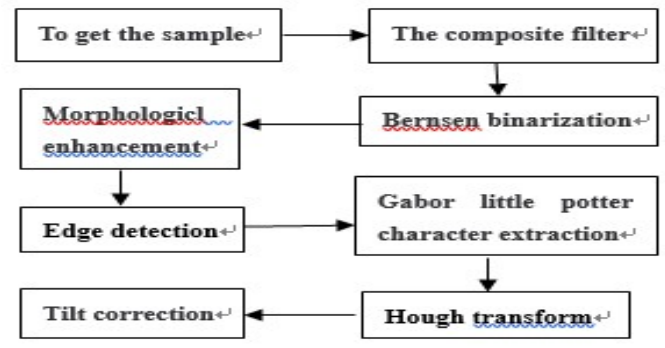

FIGURE II PREPROCESSING FLOW CHART

\section{A. Binarization}

According to the characteristics of the coded image,Bernsen method which has better adaptability to low contrast coding images is used for binarization. Its core is in pixels $(i, j)$ as the center of the window $(2 \omega+1) \times\left(2 \omega^{+1}\right)$, computing image threshold of each pixel point $T(i, j)$.

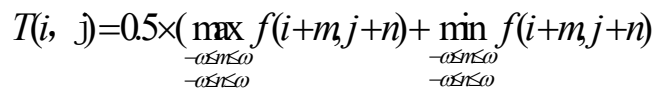

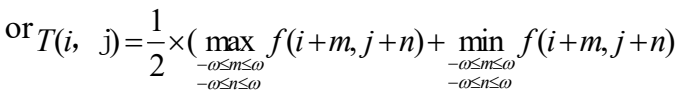

Traversal the matrix $f(i, j)$, the current grey value compared with $T(i, j)$, if more than the sentence to 1 , if less than the sentence to 0 ,record the value with matrix.

\section{B. Filter}

Screen coding images exist a variety of noise interference, the most important is the salt-and-pepper noise and Gaussian noise. After filtering it not only to achieve noise filtering at the same time to ensure that the details of the image to retain rich. Therefore, this paper proposes a combinational compression perception and morphological filtering algorithm. Firstly, the method of compression sensing is used to process the image and realize efficient filtering. And then based on the morphology that will achieve image enhancement and detail processing.

The noisy image can be expressed mathematically as

$$
\hat{x}=x+n
$$

Where $\hat{x}$ is a noisy image, $x$ for the original image, $n$ for noise. At the same time multiplied to (17) by the measurement matrix $\Phi$,

$$
y=\Phi \hat{x}=\Phi(x+n)=\Phi \Psi s=A s
$$

Solve the sparse table of the original image

$$
\min \|s\|_{1} \text { s.t. }\|A s-y\|_{2} \leq \varepsilon
$$

for(19) with Lagrange multiplication can be equivalent to deformation

$$
\min _{s}\left\{\frac{1}{2}\|A s-y\|_{2}^{2}+\lambda\|s\|_{1}\right\}
$$

Which $\lambda>0$ is the Lagrangian factor. And then restore the original image by $x=\Psi^{T} S$.

Equation (20) is the established model, and the fast iterative shrinkage threshold algorithm is used to solve the model. Iterative operator:

$$
\begin{gathered}
p_{L}(y)=\arg \min _{x}\left\{g(x)+\frac{L}{2}\left\|x-\left(y-\frac{1}{L} \nabla f(y)\right)\right\|^{2}\right\} \\
x_{k}=p_{L}\left(x_{k-1}\right)
\end{gathered}
$$

Where $\mathrm{L}$ is the step size.

consider

$$
\min _{x \in \square n}\left\{\lambda\|x\|_{1}+\frac{1}{2}\|x-y\|^{2}\right\}
$$

Equivalent to 


$$
\min _{x_{i} \in \square}\left\{\sum_{i=1}^{n}\left(\lambda\left|x_{i}\right|+\frac{1}{2}\left(x_{i}-y_{i}\right)^{2}\right)\right\}
$$

The optimal solution of $\min _{x_{i} \in \llbracket}\left\{\lambda\left|x_{i}\right|+\frac{1}{2}\left(x_{i}-y_{i}\right)^{2}\right\}$ from one dimension is

$$
\bar{x}_{i}=\operatorname{sgn}\left(y_{i}\right) \max \left\{\left|y_{i}\right|-\lambda, 0\right\}
$$

So the solution of the multidimensional case:

$$
\bar{x}=\operatorname{sgn}(y) \circ \max \{|y|-\lambda e, 0\}
$$

Where the symbol is defined as

$$
\begin{aligned}
& u^{\circ} v=\left(u_{1} v_{1}, u_{2} v_{2}, \cdots, u_{n} v_{n}\right)^{T} \\
& \operatorname{sgn}(y)=\left(\operatorname{sgn}\left(y_{i}\right)\right)_{n \times 1} \\
& |y|=\left(\left|y_{i}\right|\right)_{n \times 1}, \quad e=[1,1, \cdots, 1]^{T} \in \square^{n} .
\end{aligned}
$$

Therefore, in the solution of denoising model, Assuming that $\left\{x_{k}\right\}$ is an iterative sequence generated by the iterative algorithm

$$
\begin{aligned}
x_{k} & =p_{L}\left(x_{k-1}\right)=\arg \min _{x}\left\{g(x)+\frac{L}{2}\left\|x-\left(x_{k-1}-\frac{1}{L} \nabla f\left(x_{k-1}\right)\right)\right\|^{2}\right\} \\
& =\arg \min _{x}\left\{\lambda\|x\|_{1}+\frac{L}{2}\left\|x-x_{k-1}+\frac{1}{L} A^{T}\left(A x_{k-1}-b\right)\right\|^{2}\right\}
\end{aligned}
$$

By (26) get

$$
x_{k+1}=\operatorname{sgn}\left(y_{k}\right) \circ \max \left\{\left|y_{k}\right|-\lambda / L, 0\right\}
$$

where $L=\lambda_{\text {max }}\left(A^{T} A\right)$.

$$
\min _{x \in \square^{n}}\left\{\lambda\|x\|_{1}+\frac{1}{2}\|x-y\|^{2}\right\}
$$

Define the structure $2 \times 2$ element $w,(10)$ and (11) available:

$$
\begin{aligned}
& H a=I-(I \circ w) \\
& H b=I-(I \bullet w) \\
& J=I+H a-H b
\end{aligned}
$$

Where $\mathrm{J}$ denotes the image after morphological enhancement; Ha denotes the high-hat filtering operation on the original image; and $\mathrm{Hb}$ denotes the low-hat filtering operation on the original image.

\section{Specific algorithmic process}

a. sparse representation of the noise image

b.solve the model with a fast iterative contraction threshold algorithm

c. enter the gray scale image I to be enhanced

d. the original image, respectively, for high-hat filtering and low-hat filtering,

e. re-use (30) to achieve morphological contrast enhancement, to deal with the image $\mathrm{J}$.

\section{C.Coded Image Tilt Correction}

Based on Gabol wavelet transform algorithm method of edge feature extraction, and combined with Hough transform algorithm for image Hough transform, according to the cumulative result of Hough transform processing to correct image tilt angle. Thus it effectively complete correction of tilt of the image.

\section{EXPERIMENTAL RESULTS AND ANALYSIS}

The coding images is applied to the above basic principle for preprocessing experiments. By comparing with the traditional preprocessing method (as shown below). The validity and optimization of the preprocessing method proposed in this paper are verified. The experimental results show that the pretreatment method studied in this paper is more suitable for screen coding image preprocessing.

Figure III is the actual acquisition of noise and fuzzy screen coding defect images; Figure IV (a) shows the frequency domain sparse signal recovery results. Figure V (a) shows the compression perception and morphology combined filter results. Figure V (b) shows a result of image denoising based on compression perception. Figure V (c) shows the traditional filter and morphological combination filter results. Figure V (d) shows the traditional image filtering results. Figure VI shows the tilt correction image.
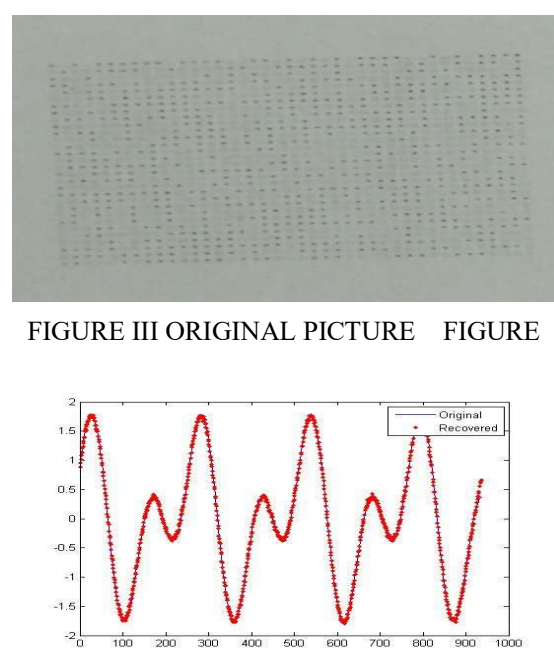

FIGURE IV (A) RECOVERY RESULT IN THE FREQUENCY DOMAIN SPARSE SIGNAL 


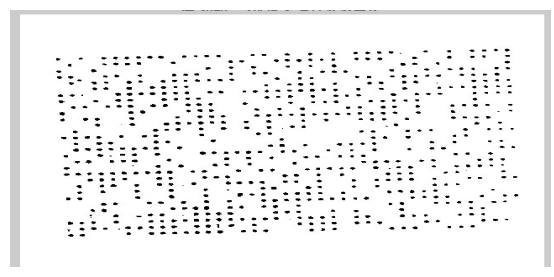

FIGURE V (A) COMPRESSION PERCEPTION AND MORPHOLOGY COMBINED FILTER RESULT

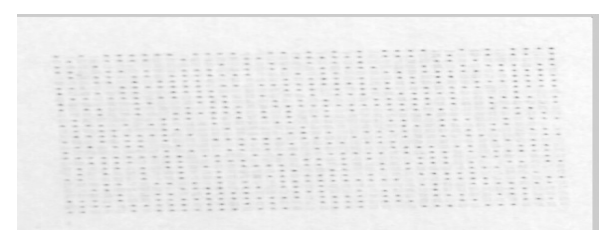

FIGURE V (B)BASED ON COMPRESSION-AWARE IMAGE DENOISING RESULT

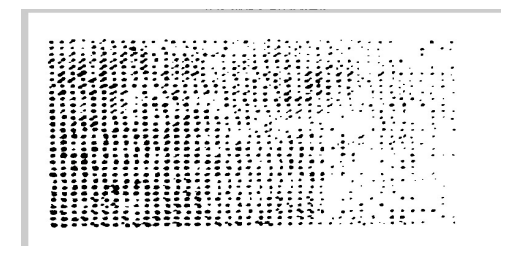

FIGURE V (C) TRADITIONAL FILTER DITIONAL FILTER AND MORPHOLOGICAL COMBINATION FILTER RESULT

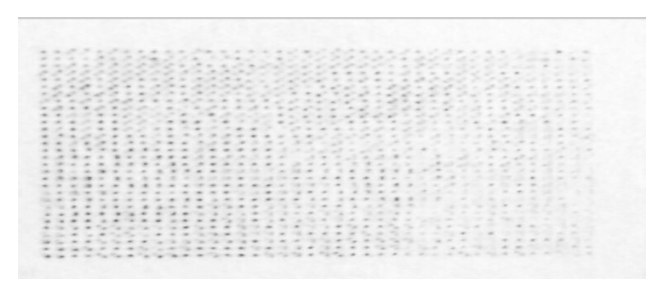

FIGURE V (D) THE TRADITIONAL IMAGE FILTERING RESULTS

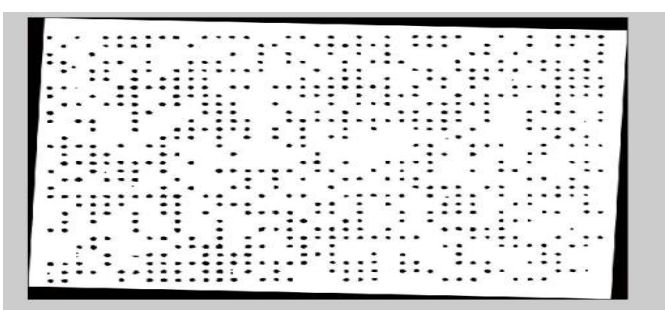

FIGURE VI TILT CORRECTION IMAGE

In order to further confirm the effectiveness of the pretreatment method, 500 different coded images were randomly collected as samples, and the pretreatment method was used to verify, statistically analyze and deal with the results. The results are shown in the figure. The experimental data show the effectiveness of the pretreatment method.

TABLE I. DIAGNOSTIC RESULTS WITH FUSION METHO

\begin{tabular}{|c|c|c|}
\hline & Pretreatment quality & Time (s) \\
\hline Sample 1 & $91.1 \%$ & 106 \\
\hline Sample 2 & $90.5 \%$ & 113 \\
\hline Sample 3 & $92.3 \%$ & 99 \\
\hline Sample4 & $94.3 \%$ & 108 \\
\hline Sample 5 & $91.6 \%$ & 116 \\
\hline
\end{tabular}

\section{CONCLUSION}

Based on the principle of compression algorithm and Combined morphology, a novel combinatorial filtering algorithm and a mesh preprocessing method based on Gobar wavelet Hough transform are combined to successfully realize the screen coding image pretreatment, and pretreatment of the image after the noise filter is obvious, the details of the information retained rich. That will expand the screen coding application areas. The experimental results show that the method is effective in the automatic recognition of screen coding and is superior to the traditional preprocessing method in practical application。

\section{ACKNOWLEDGMENT}

This work is supported partly by Natural science foundation of liaoning province under Grant No. 201602565.Professor Sun helps with sample offering and analysis,and the Shengyang Aerospace University is for site support. The author is deeply grateful for this.

\section{REFERENCES}

[1] Yanpeng Sun, Jun Xu, Ershen Wang. Screen recognition based on morphological image processing [J] Electronic Technology Applications, 2011.6

[2] Chunxue Guo. Screen coding technology introduced [J]. Electronic technology applications, 2010,7 (1): 17-23.

[3] Jin-shan Fu, Xiukun Li.Study on Sparse Decomposition of Acoustic Vector Array DOA Estimation [J]. Journal of Harbin Engineering University, 2013.3

[4] Zhang,Junkai,Research on digital watermark algorithm based on compression perception.International Journal of Security and its Applications, 2016.10.6.17

[5] Zhang,Junkai,Research on digital watermark algorithm based on compression perception.International Journal of Security and its Applications, 2016.10.6.17

[6] Duarte M F,Eldar Y C.Strucyured compressed sensing:From theory to applications.IEEE Transation $\quad$ on Signal Processing,2011,59(9):4053-4085

[7] Xiangyu Zhang ,Xiaoyu .C_V model and mathematical morphology of infrared image processing methods [J]. Laser and Infrared, 2014,44 (1): 30-34

[8] FangWang, WeiQian, Wen-chao Li.Image edge extraction method based on mathematical morphology [J] .2015.2

[9] MeiDai. Screen coding security method [P]: China,, CN 101795342 A.2010.08.04

[10] Meiniel,William.Image denoising by adaptive Compressed Sensing reconstructions and fusions[C].From proceedings of SPIE - The International Society for Optical Engineering,v 9597,2015, Wavelets and Sparsity XVI

[11] Jin, Jianqiu Yang, Bailing; Liang, Kewei. General image denoising framework based on compressive sensing theory.Computer and Graphics,V 38,n 1,p 382-391,February 2014.

[12] Zhou,Jianhua;Zhou,Siwang.Image Reconstruction for denoising based on compressive sensing.Metallurgical and Min-ing Industry [J],y 7,n 10,p 106-112,2015

[13] Wang,Gang Wang, Zesong,Liu Jinhai.A new image denoising method based on adaptive multiscale morphological edge detection.Mathe-matical Problems in Engineering,v2017,2017 